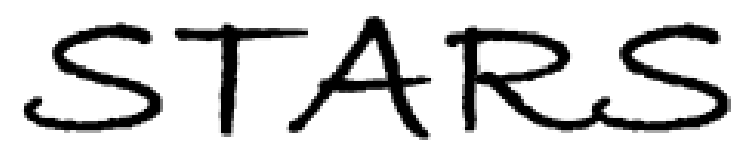

University of Central Florida

STARS

$1-1-2010$

\title{
Weak antiferromagnetic coupling in molecular ring is predicted correctly by density functional theory plus Hubbard $U$
}

\author{
Shruba Gangopadhyay \\ University of Central Florida \\ Artëm E. Masunov \\ University of Central Florida \\ Eliza Poalelungi \\ University of Central Florida \\ Michael N. Leuenberger \\ University of Central Florida
}

Find similar works at: https://stars.library.ucf.edu/facultybib2010

University of Central Florida Libraries http://library.ucf.edu

This Article is brought to you for free and open access by the Faculty Bibliography at STARS. It has been accepted for inclusion in Faculty Bibliography 2010 s by an authorized administrator of STARS. For more information, please contactSTARS@ucf.edu.

\section{Recommended Citation}

Gangopadhyay, Shruba; Masunov, Artëm E.; Poalelungi, Eliza; and Leuenberger, Michael N., "Weak antiferromagnetic coupling in molecular ring is predicted correctly by density functional theory plus Hubbard U" (2010). Faculty Bibliography 2010s. 7050.

https://stars.library.ucf.edu/facultybib2010/7050

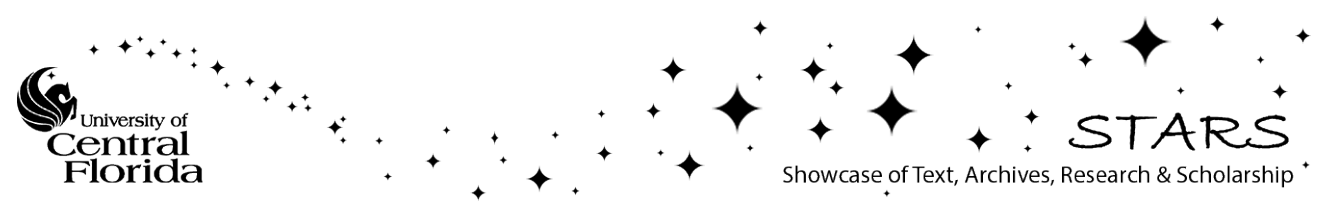




\section{Weak antiferromagnetic coupling in molecular ring is predicted correctly by density functional theory plus Hubbard $U$}

Cite as: J. Chem. Phys. 132, 244104 (2010); https://doi.org/10.1063/1.3421645

Submitted: 07 January 2009 . Accepted: 12 April 2010 . Published Online: 23 June 2010

Shruba Gangopadhyay, Artëm E. Masunov, Eliza Poalelungi, and Michael N. Leuenberger

\section{ARTICLES YOU MAY BE INTERESTED IN}

A consistent and accurate ab initio parametrization of density functional dispersion correction (DFT-D) for the 94 elements H-Pu

The Journal of Chemical Physics 132, 154104 (2010); https://doi.org/10.1063/1.3382344

Density-functional thermochemistry. III. The role of exact exchange

The Journal of Chemical Physics 98, 5648 (1993); https://doi.org/10.1063/1.464913

Perspective: How good is DFT for water?

The Journal of Chemical Physics 144, 130901 (2016); https://doi.org/10.1063/1.4944633

Where in the world is AIP Publishing? Find out where we are exhibiting next 


\title{
Weak antiferromagnetic coupling in molecular ring is predicted correctly by density functional theory plus Hubbard $\boldsymbol{U}$
}

\author{
Shruba Gangopadhyay, ${ }^{1,2}$ Artëm E. Masunov, ${ }^{1,2,3, a)}$ Eliza Poalelungi, ${ }^{1,4}$ and \\ Michael N. Leuenberger ${ }^{1,3, a)}$ \\ ${ }^{1}$ NanoScience Technology Center, University of Central Florida, 12424 Research Parkway, Suite 400, \\ Orlando, Florida 32826 USA \\ ${ }^{2}$ Department of Chemistry, University of Central Florida, 4000 Central Florida Blvd., Orlando, \\ Florida 32816 USA \\ ${ }^{3}$ Department of Physics, University of Central Florida, 4000 Central Florida Blvd., Orlando, \\ Florida 32816 USA \\ ${ }^{4}$ Department of Physics, Alexandru Ioan Cuza University, Blvd. Carol I, Nr. 11, 700506 Iasi, Romania
}

(Received 7 January 2009; accepted 12 April 2010; published online 23 June 2010)

\begin{abstract}
We apply density functional theory with empirical Hubbard $U$ parameter $(\mathrm{DFT}+U)$ to study Mn-based molecular magnets. Unlike most previous DFT $+U$ studies, we calibrate $U$ parameters for both metal and ligand atoms using five binuclear manganese complexes as the benchmarks. We note delocalization of the spin density onto acetate ligands due to $\pi$-back bonding, inverting spin polarization of the acetate oxygen atoms relative to that predicted from superexchange mechanism. This inversion may affect the performance of the models that assume strict localization of the spins on magnetic centers for the complexes with bridging acetate ligands. Next, we apply DFT $+U$ methodology to $\mathrm{Mn}_{12}$ molecular wheel and find antiparallel spin alignment for the weakly interacting fragments $\mathrm{Mn}_{6}$, in agreement with experimental observations. Using the optimized geometry of the ground spin state instead of less accurate experimental geometry was found to be crucial for this good agreement. The protocol tested in this study can be applied for the rational design of single molecule magnets for molecular spintronics and quantum computing applications. (C) 2010 American Institute of Physics. [doi:10.1063/1.3421645]
\end{abstract}

\section{INTRODUCTION}

Single molecule magnets (SMMs) attracted the interest of physicists and chemists since their initial discovery in 1993. ${ }^{1}$ Typical SMMs belong to the class of polynuclear transition metal complexes. They are characterized by a large spin ground state and considerable negative anisotropy, leading to a barrier for the reversal of magnetization. SMMs are also characterized by slow magnetization relaxation and can be magnetized below their blocking temperature. ${ }^{1}$ For these reasons, SMMs hold a great promise as potential elements of molecular spintronics and quantum computers. ${ }^{2,3}$

Spintronics is a rapidly developing area of nanotechnology, where device operation requires active manipulation of the spin degrees of freedom (in addition to the electric charge, used in traditional semiconductor electronics devices). Use of molecular elements in spintronics can take advantage of the chemical, structural, and electronic versatility provided by the molecular structures. In molecular systems, electron spins can be preserved for longer time periods and distances than in conventional inorganic materials. The low density, flexibility, transparency, processability, and novel added functionalities (magnetic switching at the molecular level, emission of light, etc.) can also be advantageous for the design of spintronic devices.

Single molecules, similar to the ones considered in this

\footnotetext{
${ }^{a)}$ Authors to whom correspondence should be addressed. Electronic addresses: amasunov@mail.ucf.edu and mleuenbe@mail.ucf.edu.
}

study, have the size c.a. $20 \AA$, and can be used as the smallest "bits" for magnetic storage and processing of information. ${ }^{4}$ Presently accessible magnetic domains have $20 \mathrm{~nm}$ in size and their further miniaturization is complicated by approaching the superparamagnetic limit. ${ }^{5}$ However, this limit does not apply to magnetic molecules because the magnetic order is determined by the electronic structure of a molecule and not by a certain critical size. In order to become practical, the intramolecular magnetic interaction have to be strong enough to prevent decoupling of the spins within the molecule by thermal fluctuations, so that the single molecule effectively behaves as an atom with a large spin $S .{ }^{4}$ Moreover, a high magnetic anisotropy is required to prevent spontaneous reorientation of the magnetization of the molecular unit, i.e., to increase its blocking temperature. ${ }^{4}$ The interaction between molecular magnetic properties and charge transport is another important issue to be considered. The combination of different properties to accomplish desirable functionality makes the rational design of the molecular magnets with optimized properties increasingly important for the spintronic applications.

The idea of using SMMs to implement the idea of quantum computer was proposed by Leuenberger and Loss. ${ }^{3}$ Instead of the classical bits, which can take only one value (1 or 0 ), quantum computers operate with quantum bits [(qubits), prepared in quantum superposition state of 1 and 0 ] and carry out multiple operations at the same time. The electron spin is a natural candidate for a qubit, as its interaction 
with environment is weaker than for the charge state. The spin can be controlled by electron spin resonance impulse in order to write, transform, and read out the information on a quantum state of the multilevel system.

Long decoherence time is critically important for the successful realization of the quantum computer. ${ }^{3,6-8} \mathrm{~A}$ specific type of molecular magnets, called antiferromagnetic (AFM) molecular wheels, attracted especial attention for this reason. ${ }^{9-12}$ Unlike other types of molecular magnets, a magnetic wheel has only one single loop of the fused transition metals polyhedra. $\mathrm{Mn}_{12}$ based wheel was first reported by Rumberger et al. ${ }^{13}$ The smallest molecule of this type is the tetranuclear manganese complex, $\left[\mathrm{Mn}_{4}(\mathrm{anca})_{4}(\mathrm{Htea})_{2}(\mathrm{dbm})_{2}\right] \cdot 2.5 \mathrm{Et}_{2} \mathrm{O}$, reported by Beedle et al. ${ }^{14}$ The larger wheels include the $\left[\mathrm{Mn}_{24}\right]$ wheel, ${ }^{15}$ the $\left[\mathrm{Mn}_{22}\right]$ wheels, ${ }^{16}$ and the $\left[\mathrm{Mn}_{84}\right]$ wheel. ${ }^{17}$ The largest spin ground state for a wheel-shaped SMM is $S=14$ for the $\left[\mathrm{Mn}_{16} \mathrm{O}_{2}\left(\mathrm{OCH}_{3}\right)_{12}(\mathrm{tmp})_{8}\left(\mathrm{CH}_{3} \mathrm{COO}\right)_{10}\right] \cdot 3 \mathrm{Et}_{2} \mathrm{O}$ wheel reported by Manoli et al. ${ }^{18}$ in 2007.

The rational design of the molecular systems for scalable quantum computer, magnetic storage, and other applications include the prediction of Heisenberg exchange constant $J$. This constant appears in Heisenberg Hamiltonian that can be written in general form as

$$
H=-\sum J_{i j} . S_{i} . S_{J} .
$$

Here, $J_{i j}$ represents the coupling constant between the two magnetic centers $i$ and $j$ with spin states $S_{i}$ and $S_{j}$. The positive $J$ values indicate the ferromagnetic ground state and the negative ones indicate the AFM ground state. For the system of two equivalent magnetic centers, the $J$ value can be calculated from the first principles using total energies of the high spin (HS) state (where $S_{i}=S_{j}$ ) and the low spin (LS) state (where $S_{i}=-S_{j}$ ).

The most common theoretical method for prediction of $J$ is broken symmetry density functional theory (BS-DFT). In this method, the energy correction is made to account for the fact that the LS state is described by the open shell single Slater determinant, which is not an eigenfunction of the spin operator (spin symmetry is "broken"). The first analytical form for the energy correction was proposed by Noodleman ${ }^{19-23}$

$$
J_{i j}=\frac{E_{\mathrm{LS}}-E_{\mathrm{HS}}}{S_{\max }^{2}}
$$

Here $E_{\mathrm{LS}}$ and $E_{\mathrm{HS}}$ are the computed energies of the LS and HS states and $S_{\max }=\left|S_{i}\right|+\left|S_{j}\right|$. This correction scheme is applicable for weakly bonded molecular fragments with small overlap between the magnetic orbitals. ${ }^{19,24,25}$ An alternative correction scheme has been used by Ruiz and co-workers. ${ }^{26}$ In this scheme, $J$ value is computed as

$$
J_{i j}=\frac{E_{\mathrm{LS}}-E_{\mathrm{HS}}}{S_{\max }\left(S_{\max }+1\right)} .
$$

This scheme assumes strong bonding between molecular fragments with localized spins, and may be more appropriate for binuclear complexes than Eq. (2). The third way to compute $J$ is independent of the bonding situation in the mol- ecule. It had been proposed by Nishino et al. ${ }^{27}$

$$
J_{i j}=\frac{E_{\mathrm{LS}}-E_{\mathrm{HS}}}{\left\langle S^{2}\right\rangle_{\mathrm{HS}}-\left\langle S^{2}\right\rangle_{\mathrm{LS}}} .
$$

Here $\left\langle S^{2}\right\rangle_{\mathrm{HS}}$ and $\left\langle S^{2}\right\rangle_{\mathrm{LS}}$ are the total spin angular momentum expectation values for high and LS states. This correction scheme approximately accounts for the overlap between magnetic orbitals. Less approximate schemes may also take into account differences in the overlap between different magnetic orbitals of the same system. ${ }^{28,29}$

Since pure DFT usually overestimates $J$ values, BS-DFT is making use of the hybrid exchange-correlation functionals, where fraction of the orbital-dependent Hartree-Fock exchange is replacing local and semi-local exchange. Unfortunately, BS-DFT was not sufficiently accurate in predictions of $J$ for binuclear complexes with acetate bridges, ${ }^{23,30}$ presumably due to strong delocalization of the spin density form the metal centers to the ligands. The attractive alternative for BS-DFT method is DFT $+U$, introduced by Anisimov et al. ${ }^{31}$ and simplified by Cococcioni et al. ${ }^{32}$ The method represents one of the simplest orbital-dependent functionals, in which a generalized Hubbard model is used to enforce localization of the electrons. A number of the first-principle approaches to estimate the $U$ parameter had been proposed. ${ }^{33,34}$ However, in many cases, quantitatively better results can be obtained with the value of $U$ determined empirically as a fitting parameter to experimental results. ${ }^{35-37}$ In this work, we adopt the empirical approach and show that both metal centers and ligand atoms need to be assigned a specific $U$ values in order to accurately describe the properties of molecular magnets.

The systems considered here belong to the general type $\mathrm{AXB}$, where magnetic centers $\mathrm{A}$ and $\mathrm{B}$ are separated by a common bridging ligand atom $\mathrm{X}$ and demonstrate the magnetic coupling by superexchange mechanism. ${ }^{38}$ The term superexchange was introduced by Anderson ${ }^{39}$ to emphasize relatively large interaction distances. His model of superexchange interaction assumes that (i) the overlap of the wave functions associated with the two magnetic centers separated by a nonmagnetic bridge is negligible; (ii) the ligand wave function is slightly modified by the presence of the magnetic ions; and (iii) this modification induces magnetic polarization in the ligand which may result in exchange interactions with other ions. Let us consider a simple example of the electron transfer from the ligand into the external shell of the magnetic ion. In the case of $d$-shells, this transfer could only take place into an empty $d$ orbital. Before interacting with the electron on the ligand orbital, each unpaired $d$-electron on each magnetic center has a spin parallel to the corresponding spin of the ligand. Both $d$-electrons interact via this bridge by an assumed AFM mechanism, thus giving rise to effectively AFM interaction of the magnetic centers. ${ }^{40}$

Considering importance of superexchange interactions, Cao et al. ${ }^{41}$ performed $\mathrm{DFT}+U$ calculations for $\mathrm{Ni}(\mathrm{hmp})(\mathrm{MeOH}) \mathrm{Cl}_{4}$ complex, where both metal and ligand oxygen atoms were assigned their $U$ parameters. Because of the strong correlation effects in this system, the pure DFT approach artificially results in the hybridization of orbitals, leading to AFM coupling. The inclusion of a Hubbard $U$ term for both the Ni $3 d$ and $\mathrm{O} 2 p$ electrons greatly enhances 
the localization, and is essential in order to obtain the correct ferromagnetic ground state and positive value for the exchange coupling constant. The rationale for using the parameter $U$ for both the $p$ and $d$ orbitals is the following. Coulomb interactions between oxygen $2 p$ electrons are comparable to those between $d$ electrons, ${ }^{42}$ and should hence be taken into consideration. However, since oxygen usually bares a fully occupied $p$-shell, this correlation effect contributes equally to the LS and HS states. Therefore, DFT $+U^{\mathrm{d}}$ already yield a satisfactory description of the ground state. However, when $2 p$ electrons of the ligand are involved in $\pi$-conjugated system, DFT $+U^{p+d}$ has to be used for both the $3 d$ and oxygen $2 p$ electrons in order to obtain the correct ground state for the molecule.

In this contribution, we predict Heisenberg exchange constant for Mn-based magnetic wheel using $\mathrm{DFT}+U$ method. We calibrate the method on four Mn(IV), Mn(III), and $\mathrm{Mn}$ (II) homovalent and one $\mathrm{Mn}(\mathrm{III})-\mathrm{Mn}$ (IV) heterovalent bimetallic complexes. This diverse benchmarking set ensured more accurate calibration of the empirical parameters than it was done in our preliminary report. ${ }^{43}$ Next, we apply this protocol to study the $\mathrm{Mn}_{12}$ (mda) AFM wheel, ${ }^{44,45}$ proposed as a molecular element for the quantum computer. The weak coupling between two parts of the wheel made possible the experimental observation of the quantum superposition involving entangled magnetic states that was reported for this system. ${ }^{46}$

\section{COMPUTATIONAL DETAILS}

All the reported calculations were done using the Quantum-ESPRESSO-4.0.1 package, ${ }^{47}$ using Perdew-BurkeErnzerhof (PBE) exchange-correlation functional, Vanderbilt ultrasoft pseudopotentials ${ }^{48}$ and a plane-wave basis set. We employ the same protocol used in our previous studies. ${ }^{49}$ Namely, the energy cutoffs for the wave functions and charge densities were set at 25 and $250 \mathrm{Ry}$ to ensure total energy convergence, spin polarized approach, the Marzari-Vanderbilt ${ }^{50}$ cold smearing (smearing factor 0.0008), and local Thomas-Fermi mixing mode to improve self consistent field (SCF) convergence. To better describe the magnetic states for manganese, both valence and semicore shells $(3 s 3 p 3 d 4 s)$ were treated explicitly, while rest of the electrons was replaced by Vanderbilt Ultrasoft pseudopotential. For homovalent AFM state, we used equal and opposite "starting magnetization" on manganese to ensure correct AFM state. For heterovalent compound, we used different starting magnetization on manganese atoms with opposite signs. All molecular structures were optimized in their most stable (LS, except for complex III) state starting from atomic coordinates, taken from x-ray diffraction data with geometrically added hydrogen atoms. The optimization was found to be critically important for the accuracy of the final results, presumably due to inaccuracies introduced in some of $\mathrm{x}$-ray structures by partial disorder.

In application of DFT $+U$ method, we followed the protocol described by Cao et al. ${ }^{41}$ We used simplified rotationalinvariant formulation, which was originally developed by Liechtenstein et al. ${ }^{51}$ as basis set independent generalization of DFT $+U$. The values of the $U$ parameter for both the metal atom and the ligand atoms $(\mathrm{O}$ and $\mathrm{N})$ were empirically adjusted to fit the experimental spin splitting energies for the benchmark set of five small binuclear manganese complexes with various oxidation states $(+2,+3$, and +4$)$, as described in Sec. III.

\section{RESULTS AND DISCUSSIONS}

In order to calibrate DFT $+U$ approach and validate it for predictions of the coupling constant values, we selected five binuclear manganese complexes presented in Fig. 1. The manganese complexes of this type have been extensively studied. They have rich redox chemistry and play a functional role in a variety of biologically important metalloproteins. ${ }^{52}$ In particular, oxomanganese clusters are either known or have been implicated in the catalytic function of manganese catalase, ${ }^{52,53}$ manganese ribonucleotide reductase, ${ }^{54}$ and the oxygen-evolving complex of photosystem II. ${ }^{55,56}$

For this work, we select a representative set of five different classes, based on the oxidation number and type of bridging groups: (I) $\mathrm{Mn}$ (IV) di- $\mu$-oxo; (II) $\mathrm{Mn}$ (IV) di- $\mu$-oxo- $\mu$-carboxylato; $\quad$ (III) $\quad \mathrm{Mn}(\mathrm{III}) \quad \mu$-oxo-di- $\mu$ carboxylato; (IV) $\mathrm{Mn}$ (II) tri- $\mu$-carboxylato; and (V) Mn(III) $\mathrm{Mn}(\mathrm{IV}) \mu$-oxo-di- $\mu$-carboxylato bridged systems. Our molecule of interest $\left[\mathrm{Mn}_{6}{ }^{\mathrm{III}} \mathrm{Mn}_{6}{ }_{6}^{\mathrm{II}}\left(\mathrm{O}_{2} \mathrm{CMe}\right)_{14}(\mathrm{mda})_{8}\right]$ (mda $=\mathrm{N}$-methyl diethanolamine) is a mixed valence Mn complex, containing both acetate and oxo bridges. We choose our benchmark molecules so that they have the similar structural features with $\left[\mathrm{Mn}_{6}{ }^{\mathrm{III}} \mathrm{Mn}_{6}{ }^{\mathrm{II}}\left(\mathrm{O}_{2} \mathrm{CMe}\right)_{14}(\mathrm{mda})_{8}\right]$. The complexes I and II represent the metal in higher oxidation state $\mathrm{Mn}(\mathrm{IV})$ bridged by oxo and acetate ligands, respectively. The complexes III and IV were selected to represent oxidation states Mn (III) and Mn(II) bridged by two and three acetate ligands, respectively, and complex $\mathrm{V}$ represents the mixed valence $\mathrm{Mn}(\mathrm{III})-\mathrm{Mn}(\mathrm{IV})$ complex, with the structure similar to the $\mathrm{Mn}(\mathrm{III})-\mathrm{Mn}(\mathrm{III})$ complex.

The exchange coupling in most of these complexes were previously studied with BS-DFT, combined with spin contamination correction schemes [Eqs. (2)-(4)]. ${ }^{23,44}$ While BSDFT gives reasonable agreement with experiment for the complex I, its prediction was $63 \%$ in error for complex II. Similar failures of hybrid DFT were reported for other molecules with acetate bridge, ${ }^{23,30}$ and were traced to the delocalization of magnetic orbitals from the manganese centers to the ligand atoms. ${ }^{23}$ For complex III, the BS-DFT not only fails to predict the quantitative value of exchange constant but also predicts the incorrect ground state. BS-DFT calculation $^{30}$ for complex $\mathrm{V}$ produced overestimated $J$ values.

Table I reports the $J$ values for the benchmark complexes, obtained in this work, and compares them to the experimental $J$ values. Predictions obtained in BS-DFT formalism using B3LYP exchange-correlation functional and spincontamination correction scheme [Eq. (2)] are also shown for comparison.

Out of the five complexes, complex IV including Mn(II) magnetic center demonstrates the weakest magnetic coupling 

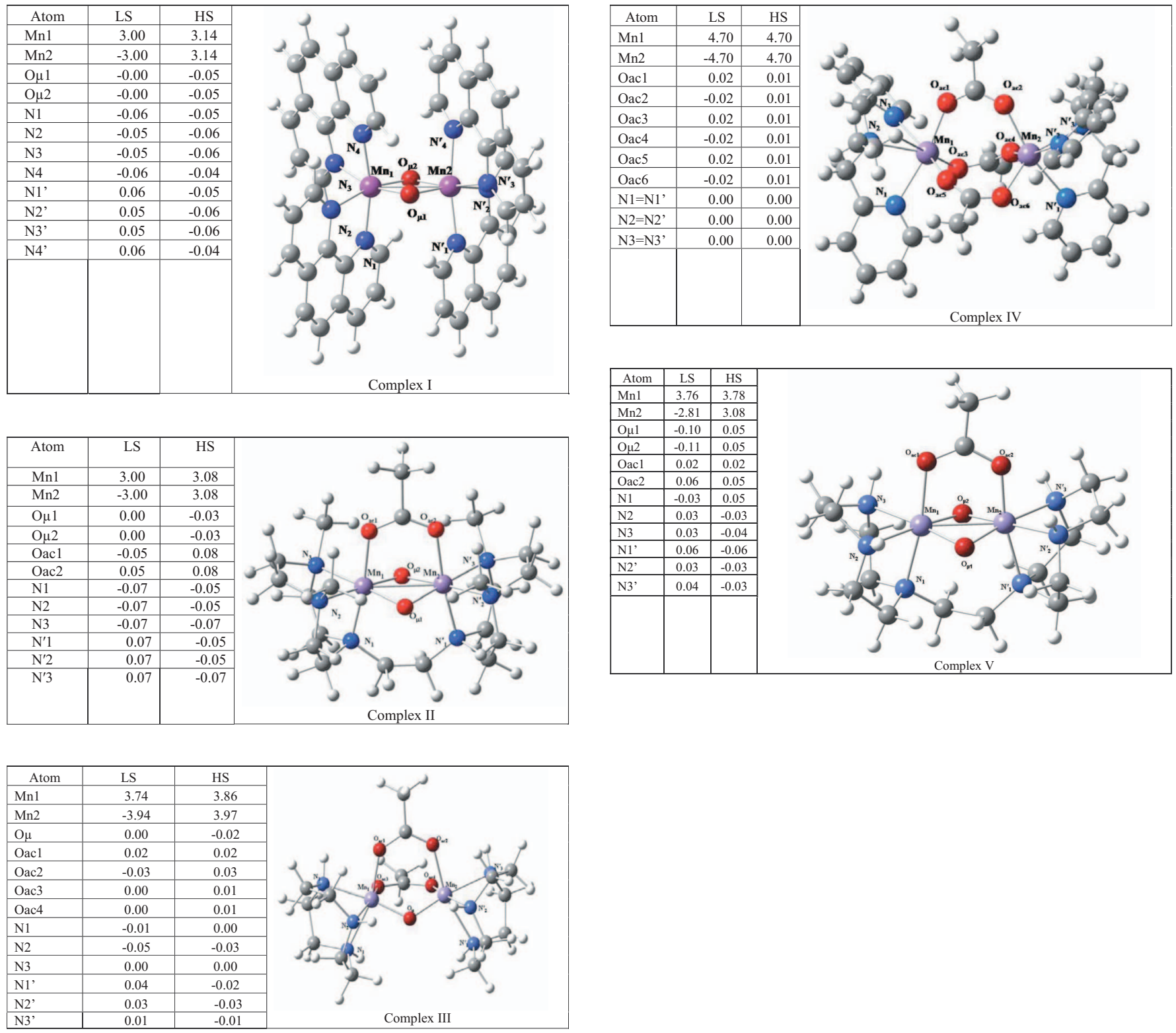

FIG. 1. Molecular structures of the binuclear complexes of the benchmark set and Lowdin spin densities in their LS and HS states.

TABLE I. Heisenberg exchange constant $J$ for the binuclear complexes, calculated using DFT $+U$ on Mn and ligands $[U(\mathrm{Mn})=2.1 \mathrm{eV}, U(\mathrm{O})=1.0 \mathrm{eV}$, and $U(\mathrm{~N})=0.2 \mathrm{eV}], \mathrm{DFT}+U$ on Mn only, and pure DFT, compared to BS-DFT predictions and to the experimental data.

\begin{tabular}{|c|c|c|c|c|c|c|}
\hline \multirow[b]{3}{*}{ Complex } & \multirow[b]{3}{*}{ Chemical formula } & \multicolumn{5}{|c|}{$\begin{array}{c}J \\
\left(\mathrm{~cm}^{-1}\right)\end{array}$} \\
\hline & & \multicolumn{3}{|c|}{ Plane wave calculations } & \multirow[b]{2}{*}{ BS-DFT } & \multirow[b]{2}{*}{ Experiment } \\
\hline & & $\begin{array}{c}\mathrm{DFT}+U \\
\text { metal+ ligand }\end{array}$ & $\begin{array}{c}\text { DFT }+U \\
\text { metal only }\end{array}$ & DFT & & \\
\hline I & {$\left[\mathrm{Mn}_{2}(\mathrm{IV})(\mu-\mathrm{O})_{2}(\text { phen })_{4}\right]^{4+}$} & -143.6 & -166.6 & -383.3 & $-131.9^{\mathrm{a}}$ & $-147.0^{\mathrm{b}}$ \\
\hline II & {$\left[\mathrm{Mn}_{2}(\mathrm{IV})(\mu \mathrm{O})_{2}((\mathrm{ac}))\left(\mathrm{Me}_{4} \mathrm{dtne}\right)\right]^{3+}$} & -71.9 & -87.4 & -255.9 & $-37.5^{\mathrm{a}}$ & $-100.0^{c}$ \\
\hline III & {$\left[\mathrm{Mn}_{2}(\mathrm{III})(\mu-\mathrm{O})(\mathrm{ac})_{2}(\operatorname{tacn})_{2}\right]^{2+}$} & 5.6 & -3.64 & -96.3 & $-40.0^{\mathrm{d}}$ & $10.0^{\mathrm{e}}$ \\
\hline IV & {$\left[\mathrm{Mn}_{2}(\mathrm{II})(\mathrm{ac})_{3}(\text { bpea })_{2}\right]^{+}$} & -7.7 & -18.8 & -13.8 & $\ldots$ & $-1.3^{\mathrm{f}}$ \\
\hline V & {$\left[\mathrm{Mn}(\mathrm{III}) \mathrm{Mn}(\mathrm{IV})(\mu-\mathrm{O})_{2}(\mathrm{ac})(\mathrm{tacn})_{2}\right]^{2+}$} & -234.0 & -247.6 & -479.3 & $-405^{\mathrm{d}}$ & $-220^{\mathrm{g}}$ \\
\hline
\end{tabular}

${ }^{\mathrm{a}}$ Reference 23.

${ }^{\mathrm{b}}$ Reference 57.

${ }^{\mathrm{c}}$ Reference 58.

${ }^{\mathrm{d}}$ Reference 30.
${ }^{\mathrm{e}}$ Reference 59.

${ }^{\mathrm{f}}$ Reference 60.

${ }^{\mathrm{g}}$ Reference 61. 
TABLE II. Dependence of Heisenberg exchange constant $(J)$ on atomic Hubbard $U$ parameters for complex II.

\begin{tabular}{lccc}
\hline \hline & $\begin{array}{c}\mathrm{U} \\
(\mathrm{eV})\end{array}$ & \multicolumn{1}{c}{$\begin{array}{c}J \\
\left(\mathrm{~cm}^{-1}\right)\end{array}$} \\
\hline $\mathrm{Mn}$ & $\mathrm{O}$ & $\mathrm{N}$ & -147.77 \\
1 & 1 & 0.2 & -71.92 \\
2.1 & 1 & 0.2 & -13.84 \\
3 & 1 & 0.2 & 48.76 \\
4 & 1 & 0.2 & 169.84 \\
6 & 1 & 0.2 & -55.27 \\
2.1 & 3 & 0.2 & -50.80 \\
2.1 & 5 & 0.2 & -62.03 \\
2.1 & 1 & 2.0 & \\
\hline \hline
\end{tabular}

$J$, similar to that in the $\mathrm{Mn}_{12}$ wheel. ${ }^{44}$ The second weakest coupling is found in complex III with $\mathrm{Mn}(\mathrm{III})$ center; this complex has ferromagnetic ground state. Complex (V) exemplifies the case of strong AFM coupling in Mn(III)$\mathrm{Mn}(\mathrm{IV})$ center mixed valence compound.

Our calculated data are in agreement with the experimental values to within $15 \%$, for both molecules with and without acetate bridge, compared to $65 \%$ maximum deviation produced by broken symmetry DFT. As one can see from this table, pure DFT dramatically overestimates the AFM interactions for all the complexes, including complex III which is known to be ferromagnetic. DFT $+U^{d}$ has good agreements with experimental results for all the complexes except complex III. DFT $+U^{p+d}$ improves the agreement with experiment for complex IV, and is the only method to reproduce the ferromagnetic ground state in complex III.

Next, we analyzed the sensitivity of the magnetic coupling to the variations in the Hubbard $U$ parameter and reported the results for the complex II in Table II. As one can see, $J$ value is the most sensitive to the choice of $U$ on $\mathrm{Mn}$ atom, with larger values drastically stabilizing HS state. Increase in the Hubbard parameter on the ligands (both $\mu$-oxo and $\mu$-carboxylato) also helps stabilize ferromagnetic state, to a smaller degree. This is in agreement with Table I where ferromagnetic state is stabilized in the order pure DFT $<\mathrm{DFT}+U^{d}<\mathrm{DFT}+U^{p+d}$.

Finally, we analyzed the electronic structure of the converged HS and LS states. The atomic spin densities obtained with Löwdin population analysis are presented in Fig. 1. As one can see from these values, the oxide dianions $(\mathrm{O} \mu)$, and aliphatic $\mathrm{N}$ atoms that serve as pure $\sigma$-donors, have spin polarization opposite to that of the nearest $\mathrm{Mn}$ ion, in agreement with superexchange mechanism we described earlier. The aromatic $\mathrm{N}$ atoms have nearly zero spin polarization. On the other hand, the $\mathrm{O}$ atoms of the acetate cations have the same spin polarization as the nearest Mn cations. This observation contradicts simple superexchange picture and can be explained with dative (also known as $\pi$-back bonding) mechanism. ${ }^{62}$ The acetate has vacant $\pi$-orbital extended over three atoms, and can serve as $\pi$-acceptor for the $d$-electrons of the Mn cation. As a result, Anderson's picture of superexchange mechanism, developed for $\sigma$-bonding metal-ligand interactions, no longer holds. This $\pi$-delocalization may be

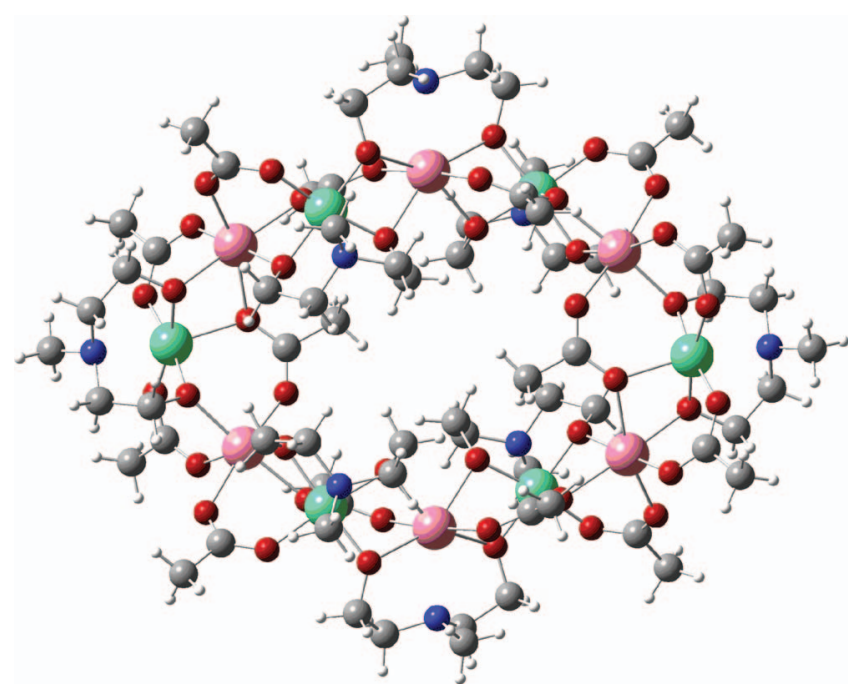

FIG. 2. Magnetic wheel complex $\left[\mathrm{Mn}_{12}\left(\mathrm{O}_{2} \mathrm{CMe}\right)_{14}(\mathrm{mda})_{8}\right]$. Pink balls denote $\mathrm{Mn}(\mathrm{III})$ and green ones denote $\mathrm{Mn}(\mathrm{II})$.

the reason why BS-DFT approach yields large numerical errors for the complexes containing acetate. The DFT $+U$ scheme, however, does not relay on this assumption and handles these acetate-containing complexes equally well.

The $U$ values for $\mathrm{Mn}, \mathrm{N}$, and $\mathrm{O}$ atoms, adjusted to reproduce the magnetic coupling constants in the binuclear complexes are reported in Table I. We applied the same approach to the $\mathrm{Mn}_{12}$ wheel polynuclear complex $\left[\mathrm{Mn}_{6}{ }^{\mathrm{III}} \mathrm{Mn}_{6}{ }^{\mathrm{II}}\left(\mathrm{O}_{2} \mathrm{CMe}\right)_{14}(\mathrm{mda})_{8}\right]$ (Fig. 2). The $\mathrm{Mn}_{12}$ wheel has two types of magnetic centers with different coordination: the $\mathrm{Mn}(\mathrm{III})$ is hexacoordinated and $\mathrm{Mn}(\mathrm{II})$ is pentacoordinated. Their spin arrangements, identified in the combined experimental and theoretical study ${ }^{44}$ are shown in Fig. 3. The theoretical BS-DFT component of that study, however, predicted a weak $\left(J=+0.04 \mathrm{~cm}^{-1}\right)$ ferromagnetic coupling instead of the correct AFM ordering for Mn1'-Mn6 (and Mn1-Mn6') centers.

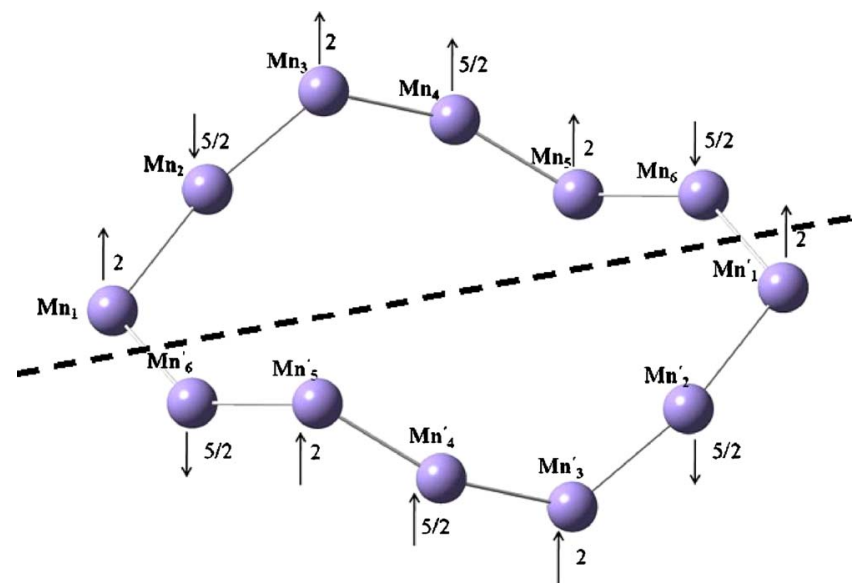

FIG. 3. The Mn-Mn distances [x-ray experiment (Ref. 44) and optimized in this work] and the spin alignments in the $S=7$ ground state of the magnetic wheel complex $\left[\mathrm{Mn}_{12}\left(\mathrm{O}_{2} \mathrm{CMe}\right)_{14}(\mathrm{mda})_{8}\right]$, with the $\mathrm{Mn} 1-\mathrm{Mn6}^{\prime}$ and Mn1'-Mn6 interactions being weakly AFM. The dashed line separates the two $S=7 / 2$ fragments that are coupled by the interactions between Mn1-Mn6' and Mn1'-Mn6; if these interactions are AFM (negative $J$ values), the resultant spin of the complete molecule is $S=7$ (Ref. 44). 
Here we preformed the geometry optimization for the LS state of the $\mathrm{Mn}_{12}$ wheel. The distances between Mn centers remained within $0.03 \AA$ from the experimental x-ray geometry (Fig. 3). This geometry relaxation was however critical in predicting the $J$ parameter between two six-center fragments (Mn1-Mn6 and Mn1'-Mn6'). The magnetic coupling $J$ (the energy difference between LS and HS states) was found to be $-0.83 \mathrm{~cm}^{-1}$ that corresponds to their AFM coupling, in agreement with the experiment ${ }^{44}$ which identified an $S=7$ ground state of the $\left[\mathrm{Mn}_{6}{ }^{\mathrm{III}} \mathrm{Mn}_{6}{ }_{6}{ }^{\mathrm{II}}\left(\mathrm{O}_{2} \mathrm{CMe}\right)_{14}(\mathrm{mda})_{8}\right]$.

\section{CONCLUSIONS}

We have performed DFT and DFT $+U$ calculations for five binuclear and one $\mathrm{Mn}_{12}$ molecular magnets. For acetate ligands, we found $\pi$-delocalization of spin density from the magnetic centers, leading to contradiction with the simple superexchange mechanism and inaccuracies in the predictions by the broken symmetry hybrid DFT method. The inclusion of a Hubbard $U$ term for both the Mn $3 d$ and $\mathrm{O}, \mathrm{N} 2 p$ electrons greatly enhanced the localization of the magnetic orbitals for both high and LS states, and was essential to obtain the correct ground state and values for the exchange coupling parameter. These properties were successfully reproduced by the plane-wave DFT $+U$ calculations. The geometry optimization of the ground magnetic state was also found to be important.

\section{ACKNOWLEDGMENTS}

The authors are grateful to DOE NERSC (project m990), I2lab and Institute for Simulation and Training (IST) Stokes HPCC facility at the University of Central Florida for the generous donation of computer time. The authors would like to thank PWscf forum members for useful discussions, anonymous reviewers for their invaluable suggestions, Chao Cao for his comments regarding DFT $+U$ calculations, Zhengji Zhao and Sampayo Hong for their help with Quantum-ESPRESSO package compilation, and Angel Crotty for her help with the preparation of this manuscript.

${ }^{1}$ R. Sessoli, H. L. Tsai, A. R. Schake, S. Wang, J. B. Vincent, K. Folting, D. Gatteschi, G. Christou, and D. N. Hendrickson, J. Am. Chem. Soc. 115, 1804 (1993); R. Sessoli, D. Gatteschi, A. Caneschi, and M. A. Novak, Nature (London) 365, 141 (1993).

${ }^{2}$ J. Lehmann, A. Gaita-Arino, E. Coronado, and D. Loss, Nat. Nanotechnol. 2, 312 (2007); A. Ardavan, O. Rival, J. J. L. Morton, S. J. Blundell, A. M. Tyryshkin, G. A. Timco, and R. E. P. Winpenny, Phys. Rev. Lett. 98, 057201 (2007).

${ }^{3}$ M. N. Leuenberger and D. Loss, Nature (London) 410, 789 (2001).

${ }^{4}$ A. V. Postnikov, J. Kortus, and M. R. Pederson, Phys. Status Solidi B 243, 2533 (2006).

${ }^{5}$ E. A. Dobisz, Z. Z. Bandic, T. W. Wu, and T. Albrecht, Proc. IEEE 96, 1836 (2008)

${ }^{6}$ F. Meier and D. Loss, Phys. Rev. Lett. 86, 5373 (2001).

${ }^{7}$ M. Affronte, F. Troiani, A. Ghirri, A. Candini, M. Evangelisti, V. Corradini, S. Carretta, P. Santini, G. Amoretti, F. Tuna, G. Timco, and R. E. P. Winpenny, J. Phys. D: Appl. Phys. 40, 2999 (2007).

${ }^{8}$ J. R. Friedman, M. P. Sarachik, J. Tejada, and R. Ziolo, Phys. Rev. Lett. 76, 3830 (1996); D. Gatteschi, A. Caneschi, L. Pardi, and R. Sessoli, Science 265, 1054 (1994).

${ }^{9}$ C. Cañada-Vilalta, T. A. O'Brien, M. Pink, E. R. Davidson, and G. Christou, Inorg. Chem. 42, 7819 (2003).

${ }^{10}$ C. Cañada-Vilalta, M. Pink, and G. Christou, Chem. Commun. (Cambridge) 2003, 1240 .
${ }^{11}$ O. Waldmann, T. Guidi, S. Carretta, C. Mondelli, and A. L. Dearden, Phys. Rev. Lett. 91, 237202 (2003).

${ }^{12}$ J. Van Slageren, R. Sessoli, D. Gatteschi, A. A. Smith, M. Helliwell, R. E. P. Winpenny, A. Cornia, A.-L. Barra, A. G. M. Jansen, E. Rentschler, and G. A. Timco, Chem.-Eur. J. 8, 277 (2002).

${ }^{13}$ E. M. Rumberger, L. N. Zakharov, A. L. Rheingold, and D. N. Hendrickson, Inorg. Chem. 43, 6531 (2004).

${ }^{14}$ C. C. Beedle, K. J. Heroux, M. Nakano, A. G. DiPasquale, A. L. Rheingold, and D. N. Hendrickson, Polyhedron 26, 2200 (2007).

${ }^{15}$ R. T. W. Scott, C. J. Milios, A. Vinslava, D. Lifford, S. Parsons, W. Wernsdorfer, G. Christou, and E. K. Brechin, Dalton Trans. 26, 3161 (2006).

${ }^{16}$ M. Murugesu, J. Raftery, W. Wernsdorfer, G. Christou, and E. K. Brechin, Inorg. Chem. 43, 4203 (2004).

${ }^{17}$ A. J. Tasiopoulos, A. Vinslava, W. Wernsdorfer, K. A. Abboud, and G. Christou, Angew. Chem., Int. Ed. 43, 2117 (2004).

${ }^{18}$ M. Manoli, A. Prescimone, A. Mishra, S. Parsons, G. Christou, and E. K. Brechin, Dalton Trans. 5, 532 (2007).

${ }^{19}$ L. Noodleman, J. Chem. Phys. 74, 5737 (1981).

${ }^{20}$ L. Noodleman and D. A. Case, Adv. Inorg. Chem. 38, 423 (1992).

${ }^{21}$ L. Noodleman and E. R. Davidson, Chem. Phys. 109, 131 (1986).

${ }^{22}$ L. Noodleman and W. G. Han, J. Biol. Inorg. Chem. 11, 674 (2006).

${ }^{23}$ E. Rudberg, P. Salek, Z. Rinkevicius, and H. Aagren, J. Chem. Theory Comput. 2, 981 (2006).

${ }^{24}$ F. Neese, J. Phys. Chem. Solids 65, 781 (2004).

${ }^{25}$ T. Soda, Y. Kitagawa, T. Onishi, Y. Takano, Y. Shigeta, H. Nagao, Y. Yoshioka, and K. Yamaguchi, Chem. Phys. Lett. 319, 223 (2000).

${ }^{26}$ E. Ruiz, J. Cano, S. Alvarez, and P. Alemany, J. Comput. Chem. 20, 1391 (1999).

${ }^{27}$ M. Nishino, S. Yamanaka, Y. Yoshioka, and K. Yamaguchi, J. Phys. Chem. A 101, 705 (1997).

${ }^{28}$ S. Goel and A. E. Masunov, Lect. Notes Comput. Sci. 5545, 141 (2009).

${ }^{29}$ S. Goel and A. E. Masunov, J. Chem. Phys. 129, 214302 (2008).

${ }^{30}$ X. G. Zhao, W. H. Richardson, J. L. Chen, J. Li, L. Noodleman, H. L. Tsai, and D. N. Hendrickson, Inorg. Chem. 36, 1198 (1997).

${ }^{31}$ V. I. Anisimov, J. Zaanen, and O. K. Andersen, Phys. Rev. B 44, 943 (1991).

${ }^{32}$ M. Cococcioni and S. de Gironcoli, Phys. Rev. B 71, 035105 (2005).

${ }^{33}$ C. Tablero, J. Phys.: Condens. Matter 20, (2008).

${ }^{34}$ H. J. Kulik, M. Cococcioni, D. A. Scherlis, and N. Marzari, Phys. Rev. Lett. 97, 103001 (2006).

${ }^{35}$ T. M. Inerbaev, S. Seal, and A. E. Masunov, J. Mol. Model. (published online in March 2010).

${ }^{36}$ A. Vincent, S. Babu, E. Heckert, J. Dowding, S. M. Hirst, T. M. Inerbaev, W. T. Self, C. M. Reilly, A. E. Masunov, T. S. Rahman, and S. Seal, ACS Nano 3, 1203 (2009).

${ }^{37}$ S. Babu, R. Thanneeru, T. Inerbaev, R. Day, A. E. Masunov, A. Schulte, and S. Seal, Nanotechnology 20, 085713 (2009).

${ }^{38}$ J. Curély, Monatsch. Chem. 136, 1013 (2005).

${ }^{39}$ P. W. Anderson and H. Hasegawa, Phys. Rev. 100, 675 (1955).

${ }^{40}$ P. W. Anderson, Phys. Rev. 80, 511 (1950).

${ }^{41}$ C. Cao, S. Hill, and H.-P. Cheng, Phys. Rev. Lett. 100, 167206 (2008).

${ }^{42}$ A. K. McMahan, R. M. Martin, and S. Satpathy, Phys. Rev. B 38, 6650 (1988); J. Yoo, E. K. Brechin, A. Yamaguchi, M. Nakano, J. C. Huffman, A. L. Maniero, L. C. Brunel, K. Awaga, H. Ishimoto, G. Christou, and D. N. Hendrickson, Inorg. Chem. 39, 3615 (2000).

${ }^{43}$ S. Gangopadhyay, A. E. Masunov, E. Poalelungi, and M. N. Leuenberger, Lect. Notes Comput. Sci. 5545, 151 (2009).

${ }^{44}$ D. Foguet-Albiol, T. A. O’Brien, W. Wernsdorfer, B. Moulton, M. J. Zaworotko, K. A. Abboud, and G. Christou, Angew. Chem., Int. Ed. 44, 897 (2005).

${ }^{45}$ S. J. Shah, C. M. Ramsey, K. J. Heroux, J. R. O'Brien, A. G. DiPasquale, A. L. Rheingold, E. del Barco, and D. N. Hendrickson, Inorg. Chem. 47, 6245 (2008)

${ }^{46}$ C. M. Ramsey, E. del Barco, S. Hill, S. J. Shah, C. C. Beedle, and D. N. Hendrickson, Nat. Phys. 4, 277 (2008).

${ }^{47}$ S. Baroni, A. Dal Corso, S. de Gironcoli, P. Giannozzi, C. Cavazzoni, G. Ballabio, S. Scandolo, G. Chiarotti, P. Focher, and A. Pasquarello, code available from http://www.quantum-espresso.org.

${ }^{48}$ D. Vanderbilt, Phys. Rev. B 41, 7892 (1990).

${ }^{49}$ S. Gangopadhayay, T. Inerbaev, A. E. Masunov, D. Altilio, and N. Orlovskaya, ACS Applied Materials \& Interfaces 1, 1512 (2009).

${ }^{50}$ N. Marzari, D. Vanderbilt, A. De Vita, and M. C. Payne, Phys. Rev. Lett. 82, 3296 (1999). 
${ }^{51}$ A. I. Liechtenstein, V. I. Anisimov, and J. Zaanen, Phys. Rev. B 52, R5467 (1995).

${ }^{52}$ G. C. Dismukes, Chem. Rev. (Washington, D.C.) 96, 2909 (1996).

${ }^{53}$ M. M. Whittaker, V. V. Barynin, S. V. Antonyuk, and J. W. Whittaker, Biochemistry 38, 9126 (1999).

${ }^{54}$ A. Willing, H. Follmann, and G. Auling, Eur. J. Biochem. 175, 167 (1988); 170, 603 (1988).

${ }^{55}$ V. K. Yachandra, K. Sauer, and M. P. Klein, Chem. Rev. (Washington, D.C.) 96, 2927 (1996); R. Manchanda, G. W. Brudvig, and R. H. Crabtree, Coord. Chem. Rev. 144, 1 (1995).

${ }^{56}$ V. L. Pecoraro, M. J. Baldwin, and A. Gelasco, Chem. Rev. (Washington, D.C.) 94, 807 (1994).

${ }^{57}$ S. Pal, M. M. Olmstead, and W. H. Armstrong, Inorg. Chem. 34, 4708
(1995).

${ }^{58}$ K. O. Schäfer, R. Bittl, W. Zweygart, F. Lendzian, G. Haselhorst, T. Weyhermuller, K. Wieghardt, and W. Lubitz, J. Am. Chem. Soc. 120, 13104 (1998)

${ }^{59}$ U. Bossek, K. Wieghardt, B. Nuber, and J. Weiss, Inorg. Chim. Acta $\mathbf{1 6 5}$, 123 (1989).

${ }^{60}$ I. Romero, L. Dubois, M. N. Collomb, A. Deronzier, J. M. Latour, and J. Pecaut, Inorg. Chem. 41, 1795 (2002).

${ }^{61}$ K. Wieghardt, U. Bossek, L. Zsolnai, G. Huttner, G. Blondin, J. J. Girerd, and F. Babonneau, J. Chem. Soc., Chem. Commun. 9, 651 (1987).

${ }^{62}$ Y. Gultneh, Y. T. Tesema, T. B. Yisgedu, R. J. Butcher, G. B. Wang, and G. T. Yee, Inorg. Chem. 45, 3023 (2006). 